\title{
All-Optical Bose-Einstein Condensates in Microgravity
}

\author{
G. Condon, M. Rabault, B. Barrett, L. Chichet, R. Arguel, H. \\ Eneriz-Imaz, D. Naik, A. Bertoldi, B. Battelier, and P. Bouyer \\ LP2N, Laboratoire Photonique, Numérique et Nanosciences, \\ Université Bordeaux-IOGS-CNRS:UMR 5298, 1 rue François Mitterrand, 33400 Talence, France
}

A. Landragin

LNE-SYRTE, Observatoire de Paris, Université PSL, CNRS, Sorbonne Université, 61 avenue de l'Observatoire, 75014 Paris, France

(Dated: June 26, 2019)

\begin{abstract}
We report on the all-optical production of Bose-Einstein condensates in microgravity using a combination of grey molasses cooling, light-shift engineering and optical trapping in a painted potential. Forced evaporative cooling in a $3-\mathrm{m}$ high Einstein elevator results in $4 \times 10^{4}$ condensed atoms every $13.5 \mathrm{~s}$, with a temperature as low as $35 \mathrm{nK}$. In this system, the atomic cloud can expand in weightlessness for up to $400 \mathrm{~ms}$, paving the way for atom interferometry experiments with extended interrogation times and studies of ultra-cold matter physics at low energies on ground or in Space.
\end{abstract}

The ability to produce ultra-cold matter and macroscopic quantum systems with laser cooling and evaporation techniques [1, 2] has brought about new physics predicted by quantum mechanics. It has also enabled new tests of fundamental physics by using matter waves for precision measurements. Today, Bose-Einstein condensates (BECs) 3] are employed as complex tools to test condensed matter phenomena, such as mimicking electronic solid state systems with atoms trapped in optical potentials [4. Producing and controlling ultra-cold matter has also lead to ground-breaking methods for measuring inertial effects [5], time and frequency standards [6], and fundamental physical constants [7] with high precision. This quantum toolbox opens exciting new prospects for inertial navigation [8, geodesy [9, tests of general relativity [10, and the detection of gravitational waves [1] and dark energy 12 .

For all of these applications, gravity can be the ultimate limiting factor. The extremely low temperatures required to study some quantum phases of matter such as antiferromagnetism [13] are usually limited by gravitational sag. Temperatures of a few nK or below also correspond to very low energy and require long observation times hardly attainable under gravity. Finally, matterwave interferometers require extended interrogation time 14] to increase their sensitivity. Circumventing gravity can be achieved by using external forces to compensate the downward pull [15, 16] or to levitate the atoms [17. In each case, the additional magnetic or optical fields used to launch or hold the atoms against gravity results in residual perturbations. Moreover, when a magnetic field is used to support neutral atoms against the gravitational force, there is always a residual curvature which limits the adiabatic decompression of the trap [18]. Extended freefall times can be achieved by launching atoms upward in an atomic fountain [19. Another method consists of letting the whole experiment fall freely under gravity, e.g. in a drop tower [20, during parabolic flight in an aircraft 21] or a sounding rocket [22], or on the International Space Station [23. Until now, BEC production on these

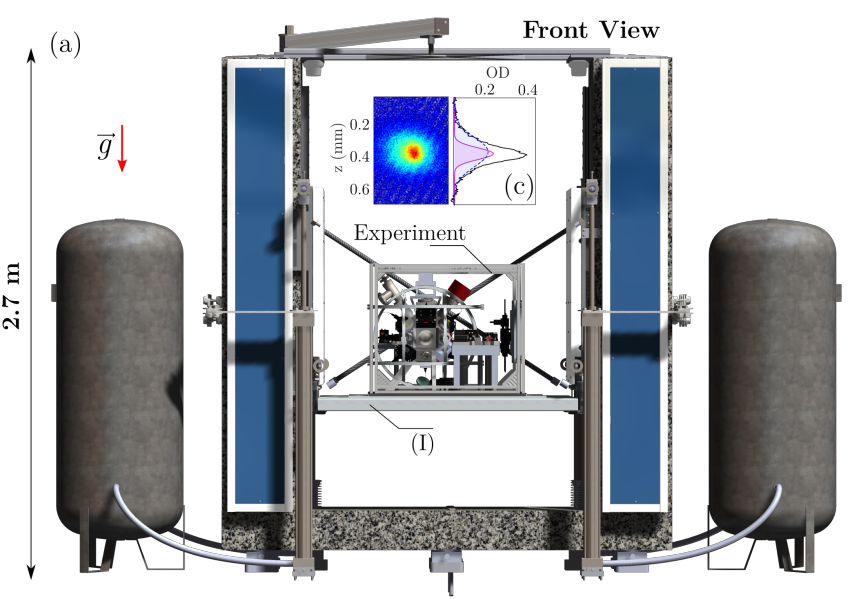

(b)

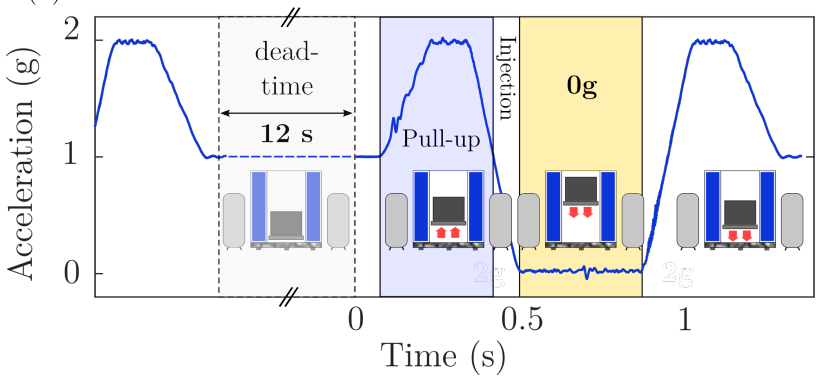

FIG. 1. (a) Schematic of the Einstein elevator. The payload (I), including the science chamber, cooling beam optics and imaging system, is driven vertically along two air-bearing translation stages. (b) Acceleration profile of the science chamber for sequential parabolas of $400 \mathrm{~ms}$, separated by a dead time of $12 \mathrm{~s}$ required to let the motors cool down. (c) Absorption image of the BEC transition after a time-of-flight of $50 \mathrm{~ms}$ in $0 \mathrm{~g}$. The projection of the vertical axis shows the typical double structure of the cloud. The blue (violet) curve is a least-squares fit to the thermal distribution (condensed fraction).

platforms has been realized only with magnetic traps using atom chip technology. 


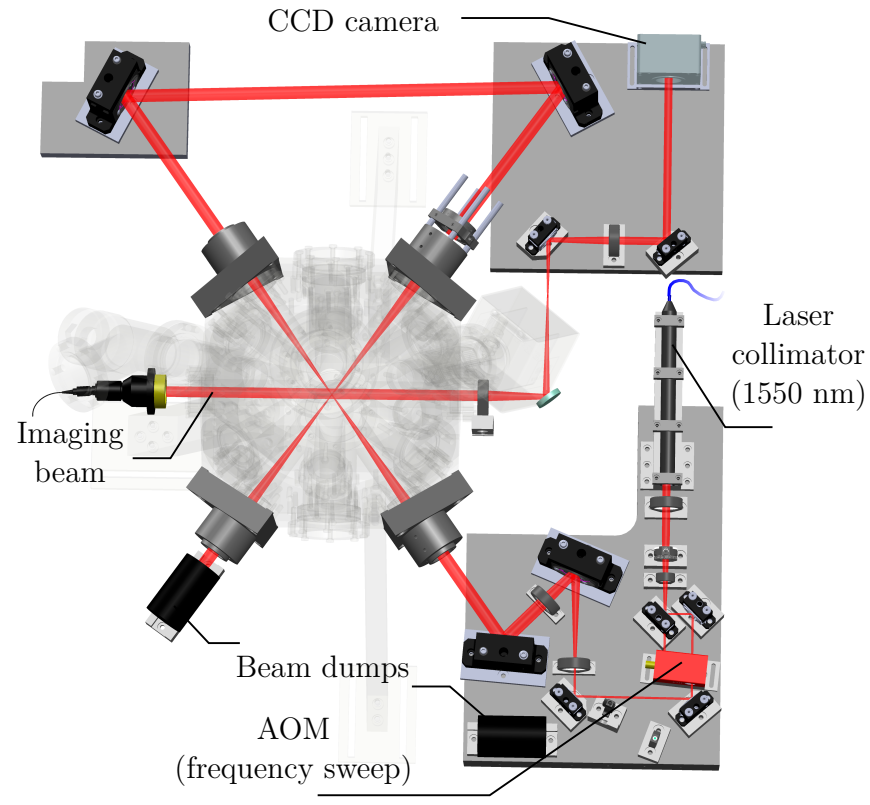

FIG. 2. Details of the painted optical trap composed of two crossed beams and the imaging system.

In this letter, we present an alternative method where ${ }^{87} \mathrm{Rb}$ BECs are produced all-optically on an Einstein elevator, as shown in Fig. 1. Our method relies on the combination of three optical techniques. First, we use a $\Lambda$-enhanced grey molasses on the $5^{2} \mathrm{~S}_{1 / 2} \rightarrow 5^{2} \mathrm{P}_{3 / 2} \mathrm{D}_{2}$ transition to create a reservoir of cold atoms [24]. Second, our $1550 \mathrm{~nm}$ optical dipole trap (ODT) creates a transparency volume due to a strong light shift of the $5^{2} \mathrm{P}_{3 / 2}$ excited state that results from coupling with the $5^{2} \mathrm{D}_{5 / 2}$ state [25]. In this way, it is possible to store the atoms in the dipole trap without emission and reabsorption of near-resonant photons [26. Third, we create a time-averaged or painted potential by spatially modulating the ODT beam. This technique leads to both a high capture volume and fast evaporative cooling with a high collision rate 27. We produce a BEC in $1.4 \mathrm{~s}$ with a critical temperature of $140 \mathrm{nK}$ before the Einstein elevator reaches the microgravity $(0 \mathrm{~g})$ phase of its trajectory.

Our experimental apparatus has been previously developed for parabolic flights onboard the Novespace Zero-G aircraft [5, 21]. It comprises fiber-based lasers, an ultrastable frequency source and a titanium science chamber housed inside a magnetic shield [Fig. 1(a)]. Figure 2 shows the optical layout of the crossed ODT beams, which are derived from a $\lambda_{D}=1550 \mathrm{~nm}$ amplified fiber laser delivering up to $23 \mathrm{~W}$. After a first telescope, an acousto-optic modulator (AOM) is used to control the optical power and to spatially modulate the beams for the painted potential. A second telescope adapts the beam profile to obtain a beam waist of $w_{0}=45 \mu \mathrm{m}$ at the location of the atoms. The trap is formed by two beams crossed at an angle of $70^{\circ}$. The key advantage of the painted potential is to break the fixed power-law relationship between the trap depth $U$ and the frequencies $\omega$ by modifying the shape of the trap. We restrict the discussion to pure harmonic traps with frequencies $\omega_{y}^{2}=\omega_{y}^{2}=\frac{8 \alpha P}{\pi m w_{0}^{4}} f_{\omega}\left(h / w_{0}\right)$ and $\omega_{z}^{2}=\frac{4 \alpha P}{\pi m w_{0}^{2} z_{R}^{2}} f_{\omega}\left(h / w_{0}\right)$, and depths $U=\frac{2 \alpha P}{\pi w_{0}^{4}} f_{U}\left(h / w_{0}\right)$, where $\alpha$ is the polarizability of the atomic ground state at $\lambda_{D}, P$ is the optical power, $m$ is the atomic mass, $z_{R}=\pi w_{0}^{2} / \lambda_{D}$ is the Rayleigh length, and $h$ is the amplitude of the spatial modulation. The functions $f_{\omega}\left(h / w_{0}\right)$ and $f_{U}\left(h / w_{0}\right)$ are the fractional reduction factors due to the spatial modulation of the beam [27. The capture volume is proportional to $h^{2}$ and can be increased with a good trade-off on the trap depth $U$ due to the factor $f_{U}\left(h / w_{0}\right)$.

We first load a 3D magneto-optical trap of approximately $5 \times 10^{7}{ }^{87} \mathrm{Rb}$ atoms from background vapour in 4 s. Atoms are cooled further by shifting the cooling beam detuning to $\Delta=-140 \mathrm{MHz}$ relative to the $\mid 5^{2} \mathrm{~S}_{1 / 2}, F=$ $2\rangle \rightarrow\left|5^{2} \mathrm{P}_{3 / 2}, F^{\prime}=3\right\rangle$ transition, while keeping the repumping beam near resonance with $\left|5^{2} \mathrm{~S}_{1 / 2}, F=1\right\rangle \rightarrow$ $\left|5^{2} \mathrm{P}_{3 / 2}, F^{\prime}=2\right\rangle$. This repump frequency is derived from the cooling beam using a fibered electro-optic modulator (EOM) operating at $6.56 \mathrm{GHz}$. We then turn on the ODT with a spatial modulation amplitude of $h=100 \mu \mathrm{m}$. The frequency of the cooling beam is then shifted further to $\Delta=-240 \mathrm{MHz}$, and we tune the repump to satisfy the Raman condition between $|F=1\rangle$ and $|F=2\rangle$ at a frequency difference of $6.834 \mathrm{GHz}$. This results in $\Lambda$ enhanced cooling and efficient ODT loading - combining the effects of grey molasses and velocity-selective coherent population trapping [24].

Outside the dipole trap [28], grey-molasses cooling results from a spatially-varying light shift that allows moving atoms to undergo a Sisyphus-like cooling process until being optically pumped to a dark state $|\mathrm{DS}\rangle$. Atoms stay in $|\mathrm{DS}\rangle$ until velocity-induced motional coupling brings them back to a bright state where cooling begins again. The lifetime of $|\mathrm{DS}\rangle$, where atoms do not scatter light, sets the minimum achievable temperature. For the $|F=2\rangle \rightarrow\left|F^{\prime}=2\right\rangle$ transition, only the ground states $\left|m_{F}=0, p=0\right\rangle$ and $\left|m_{F}= \pm 2, p= \pm 2 \hbar k\right\rangle$ are coupled, where $m_{F}$ is a magnetic quantum number, $p$ is the momentum, and $k$ is the wavevector of the cooling light. Hence, the dark state can be written as the superposition of three momentum states $|\mathrm{DS}\rangle=$ $\frac{1}{\sqrt{3}}(|-2,-2 \hbar k\rangle-|0,0\rangle+|2,2 \hbar k\rangle)$. As a result of this superposition, $|\mathrm{DS}\rangle$ is very short lived which limits the minimum temperature. In the $\Lambda$-enhanced scheme, the second laser is blue detuned from $|F=1\rangle \rightarrow\left|F^{\prime}=1\right\rangle$, and is phase locked to the first via the EOM. Consequently, $|\mathrm{DS}\rangle$ will mix with states in the $F=1$ manifold, forming a more complex but longer-lived non-coupled state $|\mathrm{NC}\rangle=\left(\Omega_{H}|G\rangle-\Omega_{G}|H\rangle\right) / \sqrt{\Omega_{G}^{2}+\Omega_{H}^{2}}$ where cold atoms are stored for larger durations - enhancing the cooling effect. Here, $|G\rangle$ and $|H\rangle$ represent superpositions of different magnetic sub-levels within the $F=1$ and $F=2$ manifolds, and $\Omega_{G, H}$ are effective optical pumping rates between these levels. The specific combinations of lev- 
els depends on the relative intensities of the cooling and repumping beams, and their polarization gradient potentials 29 .

As atoms move toward the center of the ODT, the grey-molasses light becomes increasingly blue detuned from $|F=1,2\rangle \rightarrow\left|F^{\prime}=2\right\rangle$, which strongly reduces light scattering. The total power in the ODT beams during this loading phase is $10 \mathrm{~W}$. Under these conditions, the light shift of $\left|F^{\prime}=2\right\rangle$ is $-170 \mathrm{MHz}$ at the trap center, as shown in Fig. 3(a). This leaves the $|F=1,2\rangle \rightarrow\left|F^{\prime}=3\right\rangle$ transitions far off resonance enabling low temperature atoms to become trapped in the transparency volume of the ODT [see inset of Fig. 3(b)]. In principle, the ODTinduced light shifts hinder grey molasses cooling [25]. Nevertheless, in our painted potential, atoms still have a strong probability to be cooled and pumped into $|\mathrm{NC}\rangle$ because the pumping rate $(\sim 6 \mathrm{MHz})$ is much higher than the modulation frequency of $280 \mathrm{kHz}$. Consequently a large fraction (about half) of the atoms should still undergo grey molasses cooling while being in the ODT volume. The sharp features in Fig. 3(b) comply with this hypothesis. When the cooling beams are switched off, approximately $5 \times 10^{6}$ atoms remain in the painted potential with an in-trap temperature of $15 \mu \mathrm{K}$ at a depth of $120 \mu \mathrm{K}$. We evaluate the gain of the grey molasses process by comparing it with a loading sequence using a standard red molasses. In this case, because of the light shifts, the optimal detuning varies strongly with the optical power of the ODT. In contrast, the grey molasses yields a 4-fold increase in atom number compared to the red molasses, as shown in Fig. 3(b). Moreover, the grey molasses scheme is relatively insensitive to the ODT power, and it occurs on faster timescales $(<1 \mathrm{~ms})$ than red molasses $(>5 \mathrm{~ms})$ 24, even though the final temperatures are comparable. This effect is consistent with our increased loading with the grey molasses: the longer times required for red molasses cooling lead to expansion of the atoms, which lowers the central density and therefore the loading efficiency.

After the molasses and loading phases, trapped atoms remaining in $|F=2\rangle$ are pumped into the $|F=1\rangle$ manifold using light resonant with $|F=2\rangle \rightarrow\left|F^{\prime}=2\right\rangle$. The ODT is compressed by first increasing the laser power to $20 \mathrm{~W}$ in $50 \mathrm{~ms}$ with the modulation on. The modulation amplitude is then ramped down to zero over $150 \mathrm{~ms}$ while simultaneously decreasing the ODT power to $14 \mathrm{~W}$. At this stage the trap depth is $390 \mu \mathrm{K}$, the collision rate is $5000 \mathrm{~s}^{-1}$, and the trap frequencies are $\omega_{x}=2 \pi \times 1375$ $\mathrm{Hz}, \omega_{y}=2 \pi \times 1130 \mathrm{~Hz}$, and $\omega_{z}=2 \pi \times 780 \mathrm{~Hz}$. We then proceed to a forced evaporative cooling stage by decreasing the power by a factor 400 in $1.2 \mathrm{~s}$ using a sequence of three linear ramps with different slopes to take into account the reduction in collision rate. We verified the efficiency of this method by reaching a BEC for a critical temperature $T_{\mathrm{c}}=200 \mathrm{nK}$ with $10^{5}$ atoms in standard gravity (1g).

We realize BECs in microgravity by mounting our science chamber on an Einstein elevator developed by the
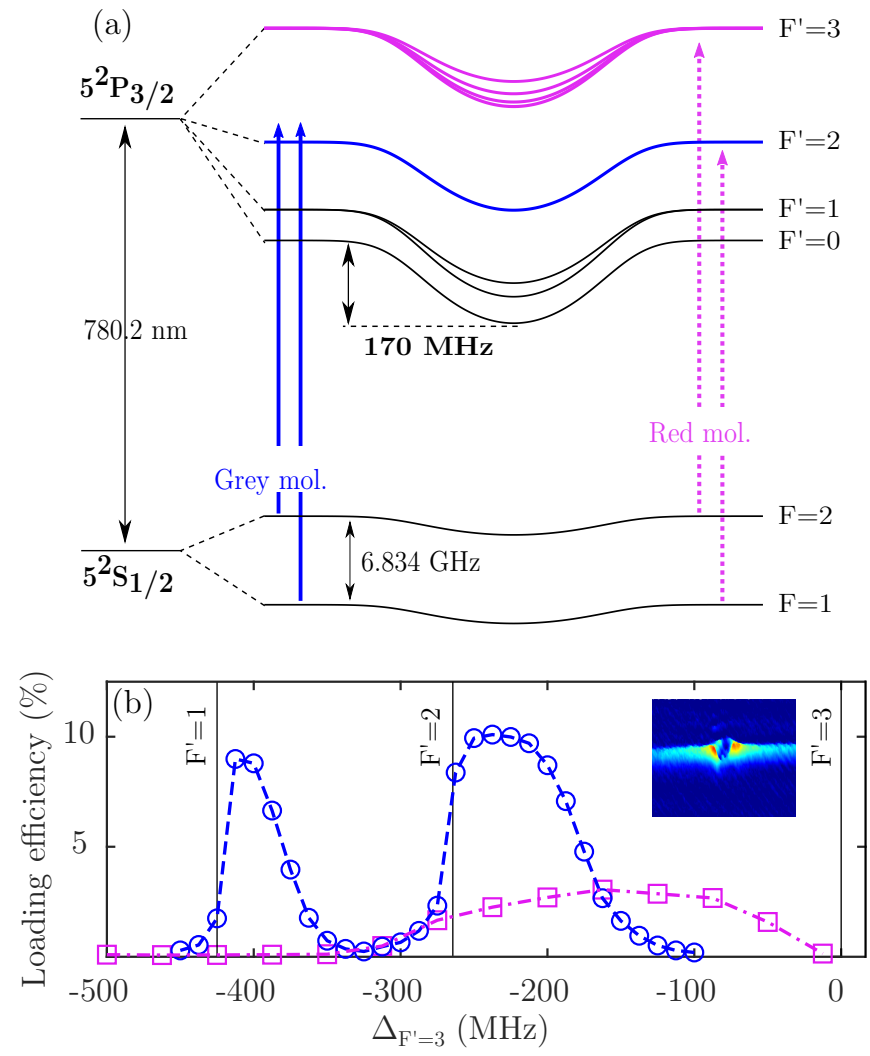

FIG. 3. (a) Level structure of the atoms including the light shift due to the painted ODT ( $P \simeq 10 \mathrm{~W}, h=100 \mu \mathrm{m})$. The cooling and repumping frequencies are shown for the grey (solid blue lines) and red (dashed pink lines) molasses. The light shift of the excited (ground) state at the center of the ODT is $\sim 170 \mathrm{MHz}(2.5 \mathrm{MHz})$. (b) ODT loading efficiency as a function of the detuning relative to $|F=2\rangle \rightarrow\left|F^{\prime}=3\right\rangle$ for the red (pink squares) and grey (blue circles) molasses. Inset: absorption image of atoms in the ODT using near-resonant light. Atoms at the center of the trap are transparent to the cooling beams.

French company Symétrie (Fig. 1), which undergoes preprogrammed parabolic trajectories that can provide up to $400 \mathrm{~ms}$ of weightlessness every 13.5 seconds. The residual acceleration during the motion, as measured with a low-noise mechanical accelerometer (Colibrys SF3600), yields a maximum amplitude of $1 \mathrm{~m} / \mathrm{s}^{2}$ and a root-meansquared repeatability of $0.05 \mathrm{~m} / \mathrm{s}^{2}$ [see Fig. 11(b)]. When operating on the elevator, the cooling sequence starts during the dead time between two parabolas, and the initial $800 \mathrm{~ms}$ of the evaporation process is the same as previously described. Just before the "pull-up" phase (when the acceleration increases above $1 \mathrm{~g}$ ), the total ODT power is $P=250 \mathrm{~mW}$ and the sample temperature is $T=400 \mathrm{nK}$. The evaporation sequence is optimized by experimentally maximizing the phase-space density at the end of the sequence. The varying acceleration during the pull-up phase strongly affects the trap depth and frequencies in time. To adapt to these changes, we apply a specific temporal profile to the ODT power (see Fig. 4). 


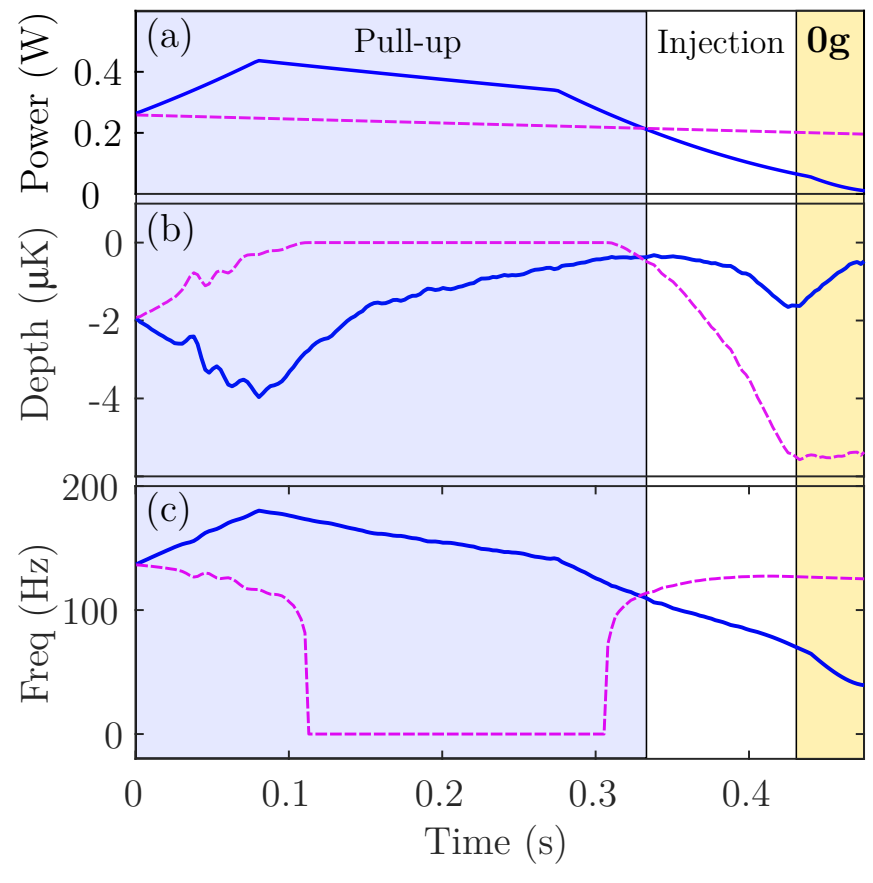

FIG. 4. Adapted ODT parameters during the pull-up, injection and $0 \mathrm{~g}$ phases (blue solid line). The parameters are calculated using the calibration of the ODT power and the measured acceleration. Calculations were validated by measuring the trap frequencies at three different times during the sequence: two in $1 \mathrm{~g}$ and one in $0 \mathrm{~g}$. (a) ODT optical power. (b) Evolution of the ODT depth. (c) Average ODT frequency taking into account the acceleration sag. For comparison, a sequence using parameters appropriate for $1 \mathrm{~g}$ (pink dashed line) shows vanishing trap depth and frequency because of the increased sag during the pull-up phase.

When the acceleration exceeds $1 \mathrm{~g}$, the trap is compressed adiabatically in $80 \mathrm{~ms}$ by increasing the power to $P=426$ $\mathrm{mW}$. This guarantees a depth sufficient (about 10 times the cloud temperature) to maintain the atoms in the trap when the acceleration reaches its maximum $(\sim 2 \mathrm{~g})$. The evaporation is completed by ramping down the power in two steps until the end of the "injection" phase (when the acceleration decreases from $1 \mathrm{~g}$ to $0 \mathrm{~g}$ ). Using this proto$\mathrm{col}$, a BEC is obtained $\sim 100 \mathrm{~ms}$ before the $0 \mathrm{~g}$ phase, with $5 \times 10^{4}$ atoms at a critical temperature of $T_{\mathrm{c}}=140$ $\mathrm{nK}$. At this point, the trap frequencies are $\omega_{x}=2 \pi \times 109$ $\mathrm{Hz}, \omega_{y}=2 \pi \times 103 \mathrm{~Hz}, \omega_{z}=2 \pi \times 71 \mathrm{~Hz}$. The total duration of the evaporation is $1.2 \mathrm{~s}$.

At the beginning of the $0 \mathrm{~g}$ phase, the ODT power is decreased in $40 \mathrm{~ms}$ to reach the minimum value required to keep the atoms in the trap. At this stage, our BEC contains $4 \times 10^{4}$ atoms for a spatial expansion corresponding to $35 \mathrm{nK}$. The ODT power is $10 \mathrm{~mW}$ for an average trap frequency of $39 \mathrm{~Hz}\left(\omega_{x}=2 \pi \times 50 \mathrm{~Hz}, \omega_{y}=2 \pi \times 41 \mathrm{~Hz}\right.$, $\omega_{z}=2 \pi \times 28 \mathrm{~Hz}$ ). We anticipate further improvements by implementing delta-kick collimation of the cloud [30]. To illustrate the importance of reducing the temperature, we produced three samples with different temperatures and measured the fluorescence signal emitted from a small detection volume as a function of the time of flight in microgravity (see Fig. 5). These data show that for $T \lesssim 100 \mathrm{nK}$ the atom number is constant inside the detection volume $\left(\sim 0.03 \mathrm{~cm}^{3}\right)$ during the full time in free-fall. For larger temperatures, atoms expand outside the detection zone and the fluorescence signal decreases dramatically.

In conclusion we have produced all-optical BECs of ${ }^{87} \mathrm{Rb}$ in microgravity with a cycle time of $13.5 \mathrm{~s}$. The painted ODT, combined with light-shift engineering and $\Lambda$-enhanced grey molasses cooling, enabled us to optimally cool and evaporate atoms to quantum degeneracy before the $400 \mathrm{~ms}$ of weightlessness available for scientific studies. These techniques can easily be adapted to other atomic species, and are compliant with sympathetic cooling for multi-species experiments. Our Einstein elevator is an alternative solution to realize experiments in microgravity as currently proposed for the ISS to study few body systems [23], new topological quantum objects such bubble-shaped traps [31, and ultra-cold atoms at extremely low temperatures [32]. Continuous operation of the elevator results in one hour of microgravity per day in a standard laboratory environment. This constitutes a new tool to prepare future Space-based missions considering ultra-cold atom interferometers for satellite gravimetry [33], quantum tests of the UFF [34, 35], and gravitational wave detection [36.

This work is supported by the following agencies: CNES (Centre National d'Etudes Spatiales), l'Agence Nationale pour la Recherche and the Délégation Générale de l'Armement under Grant "HYBRIDQUANTA" No. ANR-17-ASTR-0025-01, Grant "TAIOL" No. ANR-18QUAN-00L5-02 and Grant "EOSBECMR" No. ANR18-CE91-0003-01, the European Space Agency, IFRAF (Institut Francilien de Recherche sur les Atomes Froids), and the action spécifique GRAM (Gravitation, Relativité, Astronomie et Métrologie). M.R. thanks CNES

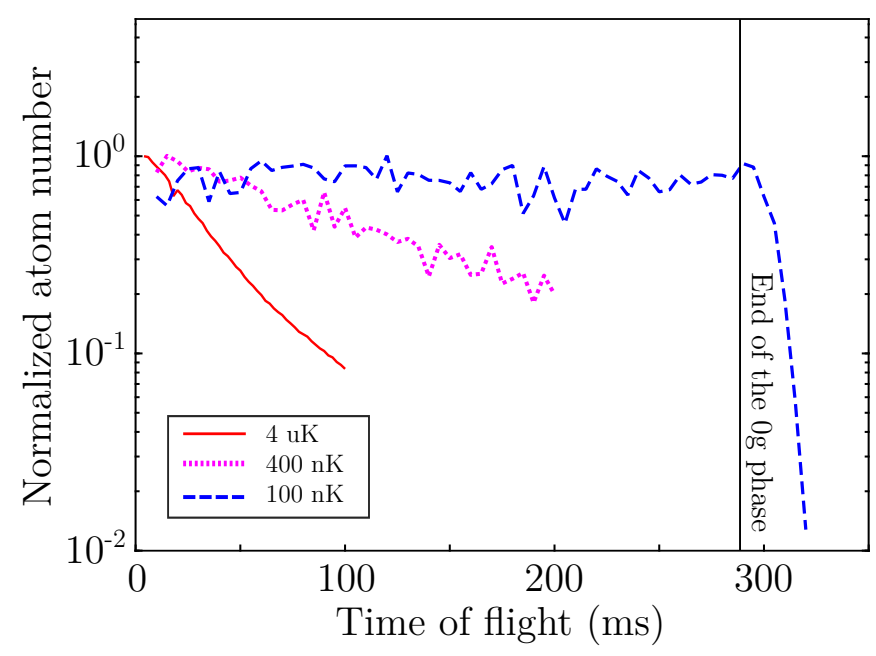

FIG. 5. Normalized atom number detected versus the time of flight in $0 \mathrm{~g}$ for three different sample temperatures. 
and IOGS for financial support. P.B. thanks Conseil

Régional d'Aquitaine for the Excellence Chair.

[1] J. Dalibard and C. Cohen-Tannoudji, "Laser cooling below the Doppler limit by polarization gradients: simple theoretical models," J. Opt. Soc. Am. B 6, 2023-2045 (1989)

[2] W. Ketterle and N. J. Van Druten, "Evaporative Cooling of Trapped Atoms," Adv. Atom. Mol. Opt. Phy. 37, 181236 (1996)

[3] M. H. Anderson, J. R. Ensher, M. R. Matthews, C. E. Wieman, and E. A. Cornell, "Observation of BoseEinstein condensate in a dilute atomic vapor," Science 269, 198-201 (1995)

[4] I. Bloch, J. Dalibard, and S. Nascimbène, "Quantum simulations with ultracold quantum gases," Nature Physics 8, 267-276 (2012).

[5] B. Barrett, P.-A. Gominet, E. Cantin, L. AntoniMicollier, A. Bertoldi, B. Battelier, P. Bouyer, J. Lautier, and A. Landragin, "Mobile and remote inertial sensing with atom interferometers," in Proceedings of the International School of Physics "Enrico Fermi", Vol. 188 "Atom Interferometry", edited by G. M. Tino and M. A. Kasevich (IOS, Amsterdam; SIF, Bologna, 2014) pp. $493-555$.

[6] A. D. Ludlow, M. M. Boyd, J. Ye, E. Peik, and P. O. Schmidt, "Optical atomic clocks," Rev. Mod. Phys. 87, 637 (2015)

7] P. Cladé, F. Nez, F. Biraben, and S. Guellati-Khelifa, "State of the art in the determination of the finestructure constant and the ratio $h / m_{u}$," C. R. Physique 20, 77-91 (2019).

[8] P. Cheiney, L. Fouché, S. Templier, F. Napolitano, B. Battelier, P. Bouyer, and B. Barrett, "NavigationCompatible Hybrid Quantum Accelerometer Using a Kalman Filter," Phys. Rev. Applied 10, 034030 (2018).

[9] A. Trimeche, B. Battelier, D. Becker, A. Bertoldi, P. Bouyer, C. Braxmaier, E. Charron, R. Corgier, M. Cornelius, K. Douch, N. Gaaloul, S. Herrmann, J. Müller, E. Rasel, C. Schubert, H. Wu, and F. Pereira dos Santos, "Concept study and preliminary design of a cold atom interferometer for space gravity gradiometry," (2019), arXiv:1903.09828

[10] C. Overstreet, P. Asenbaum, T. Kovachy, R. Notermans, J. M. Hogan, and M. A. Kasevich, "Effective Inertial Frame in an Atom Interferometric Test of the Equivalence Principle," Phys. Rev. Lett. 120, 183604 (2018).

[11] B. Canuel, A. Bertoldi, L. Amand, E. Pozzo di Borgo, T. Chantrait, C. Danquigny, M. Dovale Álvarez, B. Fang, A. Freise, R. Geiger, J. Gillot, S. Henry, J. Hinderer, D. Holleville, J. Junca, G. Lefèvre, M. Merzougui, N. Mielec, T. Monfret, S. Pelisson, M. Prevedelli, S. Reynaud, I. Riou, Y. Rogister, S. Rosat, E. Cormier, A. Landragin, W. Chaibi, S. Gaffet, and P. Bouyer, "Exploring gravity with the MIGA large scale atom interferometer," Sci. Rep. 8, 14064 (2018)

[12] B. Elder, J. Khoury, P. Haslinger, M. Jaffe, H. Müller, and P. Hamilton, "Chameleon dark energy and atom interferometry," Phys. Rev. D 94, 044051 (2016)

[13] A. Mazurenko, C. S. Chiu, G. Ji, M. F. Parsons, M. Kanasz-Nagy, R. Schmidt, F. Grusdt, E. Demler,
D. Greif, and M. Greiner, "A cold-atom Fermi-Hubbard antiferromagnet," Nature 545, 462-466 (2017).

[14] Y. Le Coq, J. A. Retter, S. Richard, A. Aspect, and P. Bouyer, "Coherent matter wave inertial sensors for precision measurements in space," Appl. Phys. B 84, 627 (2006)

[15] A. E. Leanhardt, T. A. Pasquini, M. Saba, A. Schirotzek, Y. Shin, D. Kielpinski, D. E. Pritchard, and W. Ketterle, "Cooling Bose-Einstein Condensates Below 500 Picokelvin," Science 301, 1513-1515 (2003).

[16] L. Ricci, D. Bassi, and A. Bertoldi, "Combined static potentials for confinement of neutral species," Phys. Rev. A 76, 023428 (2007)

[17] J. Billy, V. Josse, Z. Zuo, A. Bernard, B. Hambrecht, P. Lugan, D. Clément, L. Sanchez-Palencia, P. Bouyer, and A. Aspect, "Direct observation of Anderson localization of matter waves in a controlled disorder," Nature 453, 891-894 (2008)

[18] C. A. Sackett, "Limits on weak magnetic confinement of neutral atoms," Phys. Rev. A 73, 013626 (2006)

[19] T. Kovachy, P. Asenbaum, C. Overstreet, C. A. Donnelly, S. M. Dickerson, A. Sugarbaker, J. M. Hogan, and M. A. Kasevich, "Quantum superposition at the halfmetre scale," Nature 528, 530-533 (2015)

[20] T. van Zoest et. al., "Bose-Einstein condensation in microgravity," Science 328, 1540 (2010).

[21] B. Barrett, L. Antoni-Micollier, L. Chichet, B. Battelier, T. Lévèque, A. Landragin, and P. Bouyer, "Dual matterwave inertial sensors in weightlessness," Nat. Commun. 7, 13786 (2016)

[22] D. Becker et. al., "Space-borne BoseEinstein condensation for precision interferometry," Nature 562, 391-395 (2018)

[23] E. R. Elliott, M. C. Krutzik, J. R. Williams, R. J. Thompson, and D. C. Aveline, "NASAs Cold Atom Lab (CAL): system development and ground test status," npj Microgravity 4 (2018)

[24] G. Rosi, A. Burchianti, S. Conclave, D. S. Naik, G. Roati, C. Fort, and F. Minardi, " $\Lambda$-enhanced grey molasses on the D2 transition of Rubidium-87 atoms," Sci. Rep. 8, 1301 (2018).

[25] J.-F. Clément, J.-P. Brantut, M. Robert-de SaintVincent, R. A. Nyman, A. Aspect, T. Bourdel, and P. Bouyer, "All-optical runaway evaporation to BoseEinstein condensation," Phys. Rev. A 79, 061406 (2009).

[26] S. Stellmer, B. Pasquiou, R. Grimm, and F. Schreck, "Laser Cooling to Quantum Degeneracy," Phys. Rev. Lett. 110, 263003 (2013)

[27] R. Roy, A. Green, R. Bowler, and S. Gupta, "Rapid cooling to quantum degeneracy in dynamically shaped atom traps," Phys. Rev. A 93, 043403 (2016)

[28] This cooling process can also be implemented inside the dipole trap.

[29] A. T. Grier, I. Ferrier-Barbut, B. S. Rem, M. Delehaye, L. Khaykovich, F. Chevy, and C. Salomon, " $\Lambda$-enhanced sub-doppler cooling of lithium atoms in $\mathrm{D}_{1}$ gray molasses," Phys. Rev. A 87, 063411 (2013)

[30] T. Kovachy, J. M. Hogan, A. Sugarbaker, S. M. Dick- 
erson, C. A. Donnelly, C. Overstreet, and M. A. Kasevich, "Matter Wave Lensing to Picokelvin Temperatures," Phys. Rev. Lett. 114, 143004 (2015).

[31] N. Lundblad, R. A. Carollo, C. Lannert, M. J. Gold, X. Jiang, D. Paseltiner, N. Sergay, and D. C. Aveline, "Shell potentials for microgravity Bose-Einstein condensates," (2019), arXiv:1906.05885

[32] C. A. Sackett, T. C. Lam, J. C. Stickney, and J. H. Burke, "Extreme Adiabatic Expansion in Micro-gravity: Modeling for the Cold Atomic Laboratory," Microgravity Sci. Technol. 30, 155 (2018)

[33] K. Douch, H. Wu, C. Schubert, J. Müller, and F. Pereira dos Santos, "Simulation-based evaluation of a cold atom interferometry gradiometer concept for gravity field recovery," Adv. Space. Res. 61, 1307-1323 (2018)
[34] D. Aguilera et. al., "STE-QUESTtest of the universality of free fall using cold atom interferometry," Class. Quantum Gravity 31, 115010 (2014)

[35] J. Williams, S.-w. Chiow, N. Yu, and H. Müller, "Quantum Test of the Equivalence Principle and Space-Time aboard the International Space Station," New J. Phys. 18, 025018 (2016).

[36] J. M. Hogan, D. M. S. Johnson, S. Dickerson, T. Kovachy, A. Sugarbaker, S.-w. Chiow, P. W. Graham, M. A. Kasevich, B. Saif, S. Rajendran, P. Bouyer, B. D. Seery, L. Feinberg, and R. Keski-Kuha, "An atomic gravitational wave interferometric sensor in low earth orbit (AGIS-LEO)," Gen. Relativ. Gravit. 43, 1953-2009 (2011). 